\title{
Evaluation of the Shapes of Pores and Particles in Powder Metallurgy (PM) Materials Using Automated Image Analysis Techniques
}

\author{
Thomas F. Murphy
}

Research \& Development Laboratory, Hoeganaes Corporation, Cinnaminson, NJ 08077

The ability to accurately and thoroughly analyze powder metallurgy (PM) materials is heavily dependent on describing shapes of both the metallic particles used to make porous parts and the characteristics of the internal pore network. Image analysis methods, coupled with effective sample preparation procedures, have proven to be highly effective in providing a two-dimensional window into these three-dimensional microstructures.

For instance, the shape of the metallic particles is determined by the method used in their manufacture. Gas atomization produces spherical or near-spherical particles while particles produced through water atomization show more surface irregularity and roughness but are often equiaxed in shape. Both are relatively free of internal porosity. Conversely, particles produced through oxide reduction contain many internal pores, although they may also be relatively uniform in shape with an irregular surface structure.

Pore structures of sintered materials are highly dependent on the time and temperature used for sintering. Figure 1a provides an example of the irregular pore shapes resulting from lower sintering temperatures and short times while Figure $1 \mathrm{~b}$ shows smoother pore cross-sections with a lower SV of the porosity to material volume as the result of higher temperatures and longer sintering times. Mathematical expressions using combinations of linear and areal measurements can be used to analyze and compare examples of pore structures such as these. An illustration of extracted pore edges is shown in Figure 2 along with some of the measurements that can be incorporated into the expressions for making the shape comparisons.

Another use of image analysis with porous materials is in the research and development of new materials. A comparison of cross-sections from two broken tensile bars, made from materials with the same bulk chemical composition, but processed with and without ageing is shown in Figure 3. The distortion in the porosity from the tensile testing was demonstrated using localized orientation measurements at various positions from the fracture. Additionally, localized measurements of area percent porosity clearly showed an increase in porosity near the fracture due to an elongation in the pore structure in the aged material as the measurement area approached the fracture surface. Additionally, localized determinations of the bar widths could be made, verifying the yielding in the aged sample and the attendant reduction in area. Without the use of image analysis, the acquisition of these results would not have been possible. 

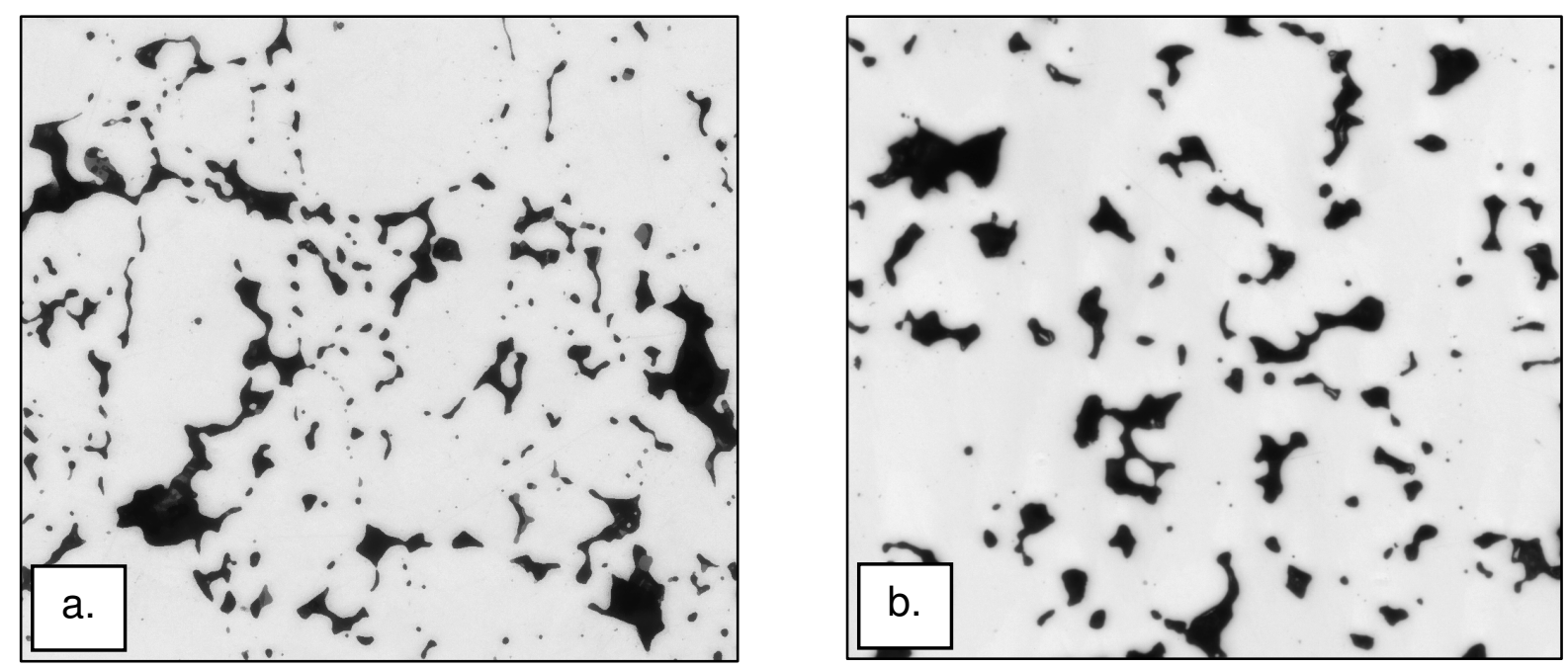

Figure 1( $a$ and $b)$. The difference in the shape of the pores is apparent in two materials sintered at a low temperature (a) and at a high temperature (b). One of the most striking differences is the loss in $S_{v}$ of the pores and particles to the material volume as the temperature is increased.

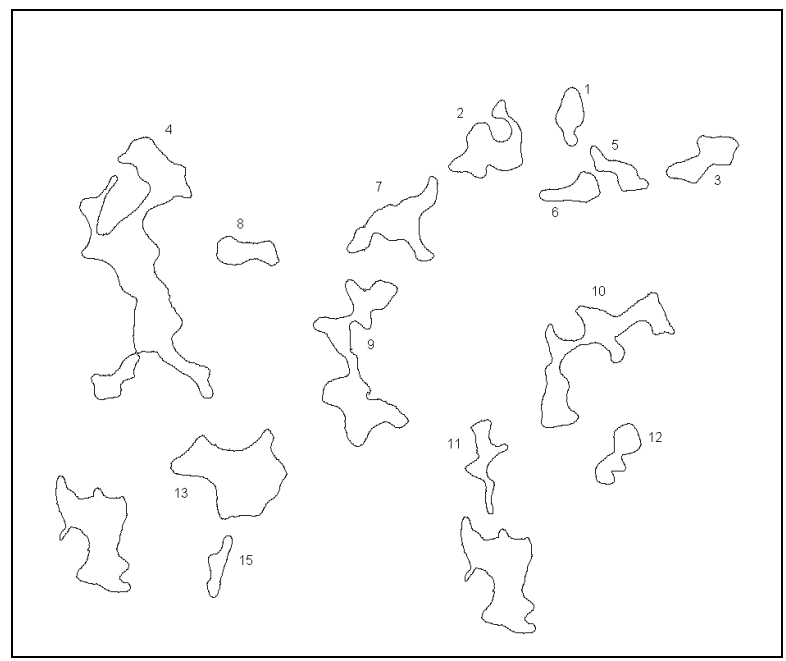

\section{Measurements used in the development of the mathematical expressions:}

- Various lengths \& widths

- Areas

- Angular projections

- Orientation

- Geometric diameters

Figure 2. Pore surfaces extracted from a sintered cross-section and examples of the measurements used in creating the mathematical basis for comparing shapes.
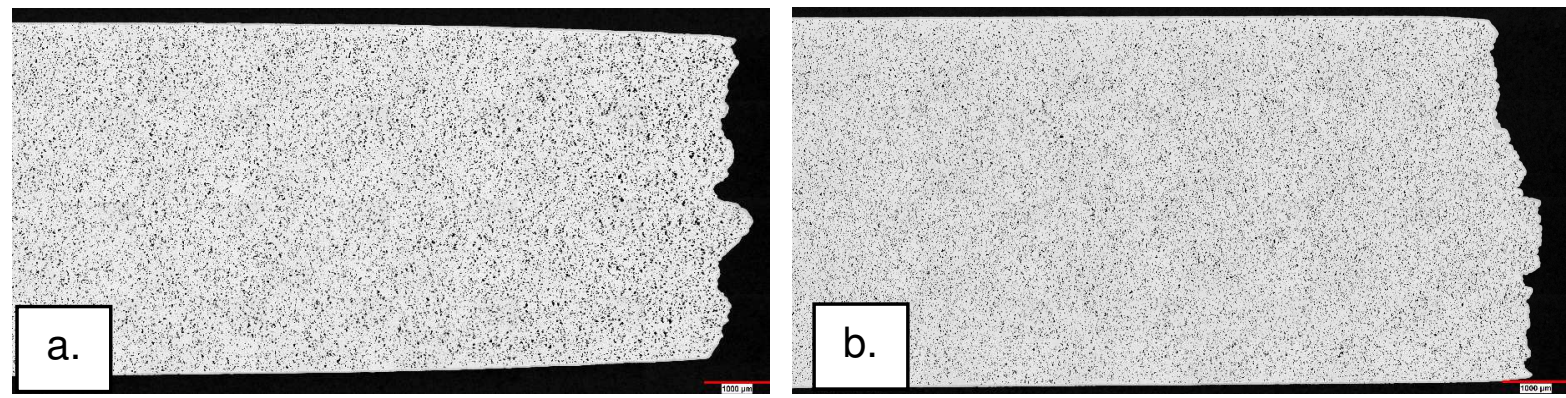

Figure 3. Cross-sectional images of two broken tensile bars. The left image (a) is the aged sample while the sample without ageing is displayed on the right (b). 